\title{
Edulcorantes no calóricos: características específicas y evaluación de su seguridad
} Non-caloric sweeteners: specific characteristics and safety assessment

\author{
Dr. Brian M. Cavagnaria
}

\section{RESUMEN}

Los edulcorantes no calóricos son aditivos alimentarios que pueden agregarse a los alimentos y bebidas, en reemplazo del azúcar, para disminuir el contenido energético y el consumo de azúcares libres. Sin embargo, muchos profesionales de la salud manifiestan varios cuestionamientos sobre la seguridad de estos aditivos para el consumo humano.

Resulta, entonces, clave quelos profesionales dela salud estén familiarizados con las características de cada edulcorante no calórico (estructura química, perfil de absorción, metabolismo y vía de excreción) y con los resultados de las minuciosas evaluaciones de su seguridad previas a su aprobación para que puedan, así, ser fuentes autorizadas de información científicamente sólida para sus pacientes.

Este artículo pretende proporcionar una mejor comprensión del proceso de evaluación de la seguridad de los edulcorantes no calóricos antes desu aprobación, locual incluye la determinación del nivel de efecto adverso no observado y de la ingesta diaria admisible.

Palabras clave: edulcorantes no calóricos, ingesta diaria admisible, seguridad.

http: / / dx.doi.org/10.5546/ aap.2019.e1

Texto completo en inglés:

a. Escuela de

Nutrición, Facultad de Ciencias Médicas, Pontificia Universidad Católica Argentina. Centro de Investigaciones Nutricionales (CINUT), Ciudad Autónoma de Buenos Aires, Argentina.

Correspondencia: Dr. Brian M. Cavagnari: bcavagna@gmail.com

Financiamiento: Ninguno.

Conflicto de intereses: Ver página e6. http:/ / dx.doi.org/10.5546/ aap.2019.eng.e1

Cómo citar: Cavagnari BM. Edulcorantes no calóricos: características específicas y evaluación de su seguridad. Arch Argent Pediatr 2019;117(1):e1-e7.

\section{INTRODUCCIÓN}

Una elevada ingesta de azúcares libres se asocia con sobrepeso y obesidad. En este contexto y con el objetivo de prevenir las enfermedades crónicas no transmisibles, la Organización Mundial de la Salud (OMS) recomienda que el consumo de azúcares libres represente menos del $10 \%$ de la ingesta calórica total diaria. Para una ingesta promedio de 2000 kcal, esto equivaldría a unos 50 gramos de azúcar diarios (18 kilos de azúcar per cápita al año). ${ }^{1}$
Sin embargo, según datos de la Organización de las Naciones Unidas para la Alimentación y la Agricultura (Food and Agriculture Organization; $\mathrm{FAO}$, por sus siglas en inglés) y de la Organización para la Cooperación y el Desarrollo Económicos (OCDE), cada persona consume un promedio anual de 24 kilos de azúcar. ${ }^{2}$

Resulta muy complejo satisfacer las preferencias de la población por el sabor dulce y cumplir con las recomendaciones de salud de la OMS en relación con los azúcares libres. Una herramienta disponible para lograr este objetivo es la utilización de edulcorantes no calóricos (ENC).

Los ENC son aditivos alimentarios que pueden agregarse a los alimentos y bebidas, en reemplazo del azúcar, para disminuir el contenido energético manteniendo la palatabilidad y el sabor dulce. ${ }^{3,4}$

Si bien todos los ENC tienen en común la capacidad de inducir percepciones de dulzor, estos compuestos son muy diversos en su estructura química (Tabla 1), perfil de absorción (Tabla 2), metabolismo (Tablas 3 y 4) y vía de excreción. Por lo tanto, es incorrecto extrapolar los efectos biológicos observados para un ENC en particular a todos los ENC en general.

Los ENC incluidos en esta revisión son algunos de los denominados edulcorantes intensivos, ya que su potencia edulcorante es varias veces superior a la de la sacarosa (Tabla 1), por lo que se requieren solo muy pequeñas cantidades en los alimentos o bebidas. ${ }^{4}$ Por lo tanto, la cantidad de ENC y de sus metabolitos absorbidos, metabolizados o excretados también 
será muy baja en comparación con la de los edulcorantes calóricos, como el azúcar.

Es importante destacar que, antes de la aprobación reglamentaria de un ENC, se realizan extensos estudios para entender el destino biológico de cada uno de ellos en el cuerpo. No obstante, y a pesar de la existencia de un importante cuerpo de evidencia sobre la seguridad de los ENC, así como de evaluaciones gubernamentales y de autoridades internacionales en materia de inocuidad de los alimentos, tanto los profesionales de la salud como la población en general manifiestan una serie de cuestionamientos sobre la seguridad de los ENC para el consumo humano. $^{7}$

Resulta, entonces, clave que los profesionales de la salud estén familiarizados con el metabolismo de los ENC y con los resultados de las minuciosas evaluaciones de su seguridad previas a su aprobación para que puedan, así, ser fuentes autorizadas de información científicamente sólida para sus pacientes.

Esta revisión tiene como objetivo principal proporcionar una mejor comprensión del

TABLA 1. Estructura química y dulzor relativo de distintos edulcorantes no calóricos

\begin{tabular}{|c|c|c|}
\hline Edulcorante no ca & Tipo de compuesto & Dulzor relativo* $^{*}$ \\
\hline Sacarina & Sulfamida de ácido benzoico. ${ }^{5}$ & 300 \\
\hline Acesulfame-k & Sal potásica del 6-metil-1,2,3 oxatiazin-4(3H)-ona-2,2-dióxido. ${ }^{6}$ & 200 \\
\hline Sucralosa & $\begin{array}{c}\text { Disacárido clorado } \\
\text { (1,6-dicloro-1,6-didesoxi- } \beta \text {-D-fructofuranosil-4-cloro-4-desoxi- } \alpha \text {-D-galactopiranósido). }{ }^{7}\end{array}$ & 600 \\
\hline $\begin{array}{l}\text { Glicósidos de } \\
\text { esteviol (estevia) }\end{array}$ & $\begin{array}{c}\text { Diterpenos glicosilados. Varios glicósidos de esteviol } \\
\text { se han aislado de las hojas de S. rebaudiana (esteviósido y rebaudiósidos } \\
\text { A, D, B y M, entre otros). Todos los glicósidos de esteviol contienen un } \\
\text { núcleo químico común, el esteviol. }\end{array}$ & $200-300$ \\
\hline Aspartamo & Éster metílico de un dipéptido compuesto por L-ácido aspártico y L-fenilalanina. ${ }^{9}$ & 200 \\
\hline
\end{tabular}

${ }^{*}$ Dulzor relativo: con relación a la sacarosa (valor $=1$ ).

TABla 2. Perfil de absorción de distintos edulcorantes no calóricos

\begin{tabular}{ll}
\hline Edulcorante no calórico & Absorción \\
\hline Sacarina & Se absorbe extensamente como molécula intacta. ${ }^{10}$ \\
Acesulfame-k & Se absorbe extensamente como molécula intacta. ${ }^{6}$ \\
Sucralosa & La mayoría $(85 \%)$ no se absorbe. El $15 \%$ se absorbe como molécula intacta. ${ }^{7}$ \\
Glicósidos de esteviol (estevia) & Solo se absorbe el metabolito esteviol proveniente de los distintos glicósidos \\
& de esteviol. ${ }^{11,12,13}$ \\
Aspartamo & El aspartamo no se absorbe como tal, sino que es digerido por completo a fenilalanina, \\
& aspartato y metanol, los cuales son, finalmente, absorbidos. ${ }^{9,14,15}$ \\
\hline
\end{tabular}

TABLA 3. Metabolismo de distintos edulcorantes no calóricos

\begin{tabular}{|c|c|}
\hline Edulcorante no calórico & Metabolismo \\
\hline Sacarina & No se metaboliza. ${ }^{10,16}$ \\
\hline Acesulfame-k & No se metaboliza. ${ }^{6,17}$ \\
\hline Sucralosa & No se metaboliza. ${ }^{7}$ \\
\hline Glicósidos de esteviol (estevia) & $\begin{array}{l}\text { Los glicósidos de esteviol no son metabolizados por las enzimas humanas, } \\
\text { sino solamente por las bacterias de la microbiota intestinal. } \\
\text { El único metabolito resultante, el esteviol, es absorbido y conjugado en el hígado } \\
\text { para facilitar su excreción. }{ }^{11-13}\end{array}$ \\
\hline Aspartamo & $\begin{array}{l}\text { Se digiere a metanol, fenilalanina y aspartato, los cuales se metabolizan de la misma } \\
\text { manera que cuando estos productos proceden de la digestión de frutas, verduras } \\
\text { y alimentos con contenido proteico. }{ }^{9,14,15}\end{array}$ \\
\hline
\end{tabular}

NOTA: En todos los casos, la eliminación es rápida, sin apreciarse bioacumulación de los ENC o de sus metabolitos en el cuerpo. $^{7}$ 
proceso de evaluación de la seguridad de los ENC más comúnmente utilizados (acesulfame-k, aspartamo, sacarina, sucralosa y glicósidos de esteviol) antes de su aprobación, lo cual incluye la determinación del nivel de efecto adverso no observado (no observed adverse effect level; NOAEL, por sus siglas en inglés) y de la ingesta diaria admisible (IDA). No se considerarán los estudios relacionados con sus efectos poscomercialización, ya que serán objeto de otra publicación.

\section{Cálculo del nivel de efecto adverso no observado}

La aprobación regulatoria de los ENC a nivel mundial se basa en la recomendación científica del Comité Mixto FAO/OMS de Expertos en Aditivos Alimentarios (Joint Expert Committee on Food Additives; JECFA, por sus siglas en inglés).

Todos los ENC utilizados en la actualidad han sido objeto de varias pruebas de seguridad muy minuciosas antes de su aprobación. ${ }^{9,19,20}$ Estas pruebas toxicológicas se realizan en animales, ya que, por cuestiones éticas, no pueden ser llevadas a cabo en seres humanos. En ellas, se emplean concentraciones crecientes del ENC por evaluar para, así, detectar los posibles efectos adversos ocasionados frente a distintas dosis del aditivo.

Las dosis más altas se utilizan para asegurar la identificación de los posibles efectos adversos. Las concentraciones menores se usan para identificar aquella dosis máxima diaria que no cause efectos adversos, también llamada "NOAEL".

Por lo tanto, el NOAEL es la cantidad de un aditivo -consumido en dosis diarias, en estudios a largo plazo- que demuestra no tener efectos adversos en los animales. ${ }^{7}$

\section{Cálculo de la ingesta diaria admisible}

Sobre el NOAEL, se aplica un factor de seguridad de 100, que es establecido por las agencias reguladoras, para considerar las diferencias por especie (los estudios se realizan en animales y no en humanos) y para asegurar la protección de los individuos más susceptibles en la población, que incluyen niños, ancianos y mujeres embarazadas. En otras palabras, la IDA se calcula dividiendo el NOAEL por 100.

Este nivel, 100 veces más bajo que el NOAEL, asegura un amplio margen de seguridad para el consumo de $\mathrm{ENC}$, ya que la ingesta máxima del aditivo que se permitirá será 100 veces menor que aquella que no causaba efectos adversos en las evaluaciones previas.

A modo de ejemplo, si la cantidad de un ENC que, en estudios con animales, mostró no tener efectos adversos -consumiéndose diariamente durante la mayoría de la vida útil del animales de $4000 \mathrm{mg}$ por kilogramo de peso corporal por día, el NOAEL será de $4000 \mathrm{mg} / \mathrm{kg}$ / día. Al aplicar un factor de seguridad de 100, la IDA será de $40 \mathrm{mg} / \mathrm{kg} /$ día.

En definitiva, para establecer la IDA, hay una reducción de 100 veces la cantidad que no mostró tener efectos adversos. Este es un factor de seguridad mucho mayor que el existente para la mayoría de los nutrientes y los componentes naturales de los alimentos. Por lo tanto, la IDA es un nivel de ingesta diaria considerado seguro para todos, entre los que se incluyen aquellos con la exposición potencial más alta a un aditivo alimentario. $^{7}$

La IDA es la herramienta más importante para poder garantizar un uso apropiado y seguro de

TABLA 4: Metabolismo de los distintos productos de digestión del aspartamo

\section{Productos de digestión del aspartamo Metabolismo}

Metanol

Aspartato

Fenilalanina
Entra en la circulación portal y se metaboliza a formaldehído por la alcoholdeshidrogenasa. Este se oxida a ácido fórmico por la formaldehído-deshidrogenasa. El ácido fórmico se excreta del cuerpo en la orina o se metaboliza a dióxido de carbono y se excreta a través de la respiración. ${ }^{9}$

Se convierte en oxalacetato por transaminación. Luego llega a la circulación portal y pasa a formar parte del reservorio de aminoácidos libres. El oxalacetato y el aspartato son interconvertidos y pueden participar en el ciclo de la urea y la gluconeogénesis. El aspartato también puede usarse para generar otros aminoácidos esenciales, como metionina, treonina, isoleucina y lisina. Finalmente, el exceso de aspartato es excretado en la orina. ${ }^{18}$

Entra por circulación portal al hígado, donde puede ser parcialmente convertida en tirosina por la fenilalanina hidrolasa. ${ }^{19}$ La fenilalanina que llega a la circulación sistémica puede ser distribuida en todo el cuerpo, incluido el cerebro, donde es necesaria para el crecimiento y desarrollo normales. ${ }^{9}$ También puede convertirse en tirosina y, finalmente, en dopamina, norepinefrina y epinefrina.

La ingesta de fenilalanina en exceso se excreta en la orina. 
los ENC. La IDA de cada ENC se puede apreciar en la Tabla 5.

El uso de la IDA para la evaluación toxicológica y de seguridad de los aditivos alimentarios es aceptado por todas las autoridades regulatorias del mundo. ${ }^{7}$

Dado que el cálculo de la IDA tiene en cuenta el consumo durante toda la vida, brinda un margen de seguridad lo suficientemente amplio como para no alarmarse si la ingesta a corto plazo de un individuo excede los niveles de la IDA, siempre que el consumo promedio a largo plazo no lo haga. ${ }^{22-25}$

\section{Exposición habitual a los edulcorantes no calóricos}

Antes de la aprobación de un ENC, la exposición potencial para aquellas personas de mayor consumo se calcula considerando los datos obtenidos de encuestas alimentarias, así como los niveles propuestos de uso de ese ENC en diversos alimentos y bebidas.

Así, se establecen niveles máximos de uso para distintas categorías de alimentos, de forma tal de asegurar que, incluso aquellas personas de mayor consumo, no excedan la IDA. El nivel habitual de consumo de ENC en la población está por debajo de la IDA. ${ }^{7}$

\section{Características de los distintos edulcorantes no calóricos}

Acesulfame de potasio (acesulfame-k)

El acesulfame-k es un ENC ampliamente utilizado, por lo general, en combinación con otros ENC. En la actualidad, está autorizado en más de 90 países.

La estabilidad del acesulfame-k en los alimentos, en especial, su resistencia a la degradación térmica, lo convierte en un ENC muy atractivo para usar en alimentos y bebidas. ${ }^{6,26}$

El acesulfame-k es un compuesto hidrofílico que, al ingerirse, se absorbe rápida y casi completamente en la circulación sistémica. ${ }^{6,17}$ Una vez absorbido, se distribuye a través de la sangre a todos los tejidos del cuerpo.

Estudios en humanos han demostrado que el acesulfame-k no se metaboliza antes de su excreción por vía renal, dentro de las 24 horas posteriores al consumo. ${ }^{6,27}$

\section{Aspartamo}

El aspartamo es un ENC ampliamente utilizado en todo el mundo como endulzante de mesa y en una amplia variedad de bebidas y alimentos.

Su contenido calórico por gramo es similar al de la sacarosa (4 calorías por gramo), pero, al solo utilizarse una muy pequeña cantidad para lograr el dulzor requerido, prácticamente, no aporta ninguna caloría en los productos que lo contienen.

El aspartamo es un ENC aprobado por el JECFA, por la Food and Drug Administration (FDA) y por otras prestigiosas organizaciones regulatorias en más de 110 países. ${ }^{18,28}$

Tras su ingesta, el aspartamo se digiere por acción enzimática (esterasas y peptidasas) en sus tres componentes principales: metanol $(10 \%$ en peso de aspartamo), ácido aspártico (40\%) y fenilalanina (50\%). Nada del aspartamo intacto llega a la circulación..$^{9,14,15}$ Solo los tres productos de digestión llegan al torrente sanguíneo y se encuentran en la misma forma que cuando se absorben a partir de fuentes de alimentos, tales como frutas, verduras y alimentos proteínicos, como carne, pescado, huevos o legumbres. ${ }^{7}$

En el cuerpo, los 3 productos de digestión siguen sus vías metabólicas normales: se absorben en los tejidos o se excretan (Tabla 4).

TABLA 5. Ingesta diaria admisible de los distintos edulcorantes no calóricos

\begin{tabular}{lc}
\hline Edulcorantes no calóricos & Ingesta diaria admisible (mg/kg peso corporal/día) \\
\hline Acesulfame-k & 15 \\
Aspartamo & 40 \\
Sacarina & 5 \\
Sucralosa & 15 \\
Glicósidos de esteviol (estevia) & $4^{*}$ \\
\hline
\end{tabular}

* Debido a que todos los glicósidos de esteviol se metabolizan a un producto metabólico final común (el esteviol), el JECFA estableció una IDA "para todos los glicósidos de esteviol" en función de la cantidad de esteviol producida por cada glicósido de esteviol luego de su hidrólisis, que denominó "equivalentes de esteviol". La IDA se fijó en $4 \mathrm{mg}$ de equivalentes de esteviol por kilogramo de peso corporal por día. ${ }^{21}$ A modo de ejemplo, esto equivaldría a una IDA de $12 \mathrm{mg} / \mathrm{kg} /$ día para el rebaudiósido A. 


\section{Sacarina}

En los humanos, la sacarina absorbida se une reversiblemente a las proteínas plasmáticas y es distribuida a través de la sangre a los órganos del cuerpo. Entre el $85 \%$ y el $95 \%$ de la sacarina absorbida se elimina por la orina. El resto se excreta en las heces. ${ }^{10,16}$

\section{Sucralosa}

La sucralosa se obtiene por halogenación selectiva de la sacarosa: se produce a partir de ella mediante el reemplazo de los grupos hidroxilo en las posiciones $4,1^{\prime}$ y $6^{\prime}$ por cloro, y se obtiene una $4,1^{\prime}, 6^{\prime}$-triclorogalactosacarosa. Este cambio da lugar a una modificación en la conformación de la molécula, de tal manera que las enzimas glucosídicas que hidrolizan la sacarosa son incapaces de escindir la sucralosa. Por lo tanto, la sucralosa no es digerida en monosacáridos ni es metabolizada para obtener energía por el cuerpo; no aporta calorías ni afecta los niveles de glucosa en la sangre. ${ }^{7}$

La sucralosa es pobremente absorbida; su metabolismo es pequeño y se excreta, sobre todo, sin cambios en las heces. ${ }^{7}$

Es estable a altas temperaturas, por lo que se convierte en un edulcorante ideal para su uso en bebidas y otros alimentos, que incluyen aquellos sometidos a tratamiento térmico, como los productos horneados.

\section{Glicósidos de esteviol (estevia)}

El extracto de hojas de estevia es un ENC derivado de la planta Stevia rebaudiana (Bertoni), que contiene compuestos de sabor dulce llamados "glicósidos de esteviol" (esteviósido, rebaudiósidos $\mathrm{A}, \mathrm{D}, \mathrm{B}, \mathrm{M}$, etc.). ${ }^{29,30}$

Las enzimas y los ácidos presentes en el tracto gastrointestinal superior no hidrolizan los glicósidos de esteviol, sino que todos son metabolizados a un producto final común, el esteviol, por las bacterias intestinales. ${ }^{11-13,31-33}$

El esteviol, en cambio, es resistente a la degradación bacteriana ${ }^{12}$ y sí es absorbido, glucuronizado en el hígado y transportado vía bilis de nuevo hacia el tracto intestinal, donde es, finalmente, excretado. ${ }^{7}$

En los humanos, el principal metabolito es el glucurónido de esteviol, que se excreta, sobre todo, por la orina. . $^{32,34}$

\section{Aspectos metabólicos de los edulcorantes no calóricos relacionados con el embarazo y la lactancia \\ Acesulfame-k}

Este ENC puede transferirse a través de la placenta y encontrarse en los tejidos fetales en bajas concentraciones..$^{35}$ Las concentraciones en la placenta son superiores a las fetales, por lo que esta actúa como barrera protectora. La exposición materna al acesulfame-k no plantea un riesgo para el feto. ${ }^{7}$

Se han reportado niveles bajos de acesulfame-k en la leche materna humana, aunque el nivel detectado está varios órdenes de magnitud por debajo de la IDA. ${ }^{36}$

\section{Aspartamo}

Debido a la rápida digestión del aspartamo en el lumen gastrointestinal y en las células de la mucosa intestinal antes de llegar al torrente sanguíneo, la molécula de aspartamo intacta nunca está presente en los tejidos internos del cuerpo ni en la leche materna., ${ }^{9,18}$

No hay datos epidemiológicos o experimentales que indiquen que el aspartamo cruce la placenta o que el consumo materno produzca efectos adversos para la madre o el feto. $^{37,38}$ De hecho, las ingestas por encima de la IDA (hasta $200 \mathrm{mg} / \mathrm{kg}$ ) no se han asociado con riesgos para la salud fetal. ${ }^{37,38}$

No se encuentra aspartamo en la leche materna de mujeres lactantes que consumieron este ENC. ${ }^{36}$

\section{Sucralosa}

No hay evidencia de que la sucralosa sea transportada a través de la placenta. ${ }^{7}$

\section{Sacarina}

Estudios en roedores han demostrado que la sacarina puede atravesar la placenta y que, debido al lento metabolismo fetal, permanece en los tejidos fetales más tiempo que en los adultos. ${ }^{37,38}$ La sacarina también puede transferirse a través de la placenta y aparecer en los tejidos fetales humanos.

Basándose en la evidencia disponible en animales y en la falta de estudios contradictorios en seres humanos, no se recomienda la sacarina para su uso durante los períodos de preconcepción y periconcepción..$^{37,38}$

\section{Ciclamato}

El ciclamato atraviesa la barrera placentaria y se encuentra en el líquido amniótico, lo que indica 
que llega a los tejidos fetales. ${ }^{39}$

No se recomienda el consumo de ciclamato durante el embarazo. ${ }^{37-39}$

Como conclusión, se puede decir que el consumo de sacarina y ciclamato no se recomienda durante el embarazo. Otros ENC (aspartamo, acesulfame-k y sucralosa) no han demostrado ser inseguros durante el embarazo si se consumen por debajo de la IDA. ${ }^{37,38}$

\section{Aspartamo y fenilcetonuria}

Al administrar $34 \mathrm{mg} / \mathrm{kg}$ de aspartamo (percentil 99 de la ingesta) a pacientes con fenilcetonuria moderada, la fenilalanina plasmática aumenta a $16 \mathrm{mmol} / \mathrm{dl}$, contra 11 $\mathrm{mmol} / \mathrm{dl}$ en los adultos sanos. ${ }^{40,41}$

La administración de dosis más bajas (10 mg/ $\mathrm{kg}$ )-que reflejan más razonablemente el consumo real- eleva el nivel plasmático de fenilalanina en individuos sanos a $6 \mathrm{mmol} / \mathrm{dl}$ (desde una línea base de $4,5 \mathrm{mmol} / \mathrm{dl}$ ), mientras que el nivel en heterocigotos para fenilcetonuria alcanza los 8 $\mathrm{mmol} / \mathrm{dl}$ (línea de base de 6,9 mmol/ dl). ${ }^{42}$

La Agencia Europea de Seguridad Alimentaria (European Food Safety Agency; EFSA, por sus siglas en inglés) concluye que, en pacientes con fenilcetonuria, la IDA del aspartamo no es aplicable, ya que requieren una adherencia estricta a una dieta baja en fenilalanina proveniente de todas las fuentes, incluido el aspartamo. ${ }^{43}$

Por ese motivo, los alimentos que contienen aspartamo deben contener en el rótulo la leyenda "Fenilcetonúricos: contiene fenilalanina" o una frase similar. ${ }^{44}$

\section{DISCUSIÓN Y CONCLUSIONES}

Muchos profesionales de la salud se refieren de forma genérica a "los ENC", como si todos se trataran de un mismo compuesto, con las mismas propiedades químicas y los mismos destinos metabólicos. Si bien los distintos ENC aportan dulzor a los alimentos y bebidas, es muy importante remarcar que todos son moléculas diferentes. Por este motivo, los potenciales efectos sobre la salud de un edulcorante no son extrapolables a los demás.

A pesar de las extensas pruebas de seguridad que se realizan antes de su aprobación y de la comprensión acabada de la absorción, distribución, metabolismo y excreción de los distintos ENC, la seguridad de estos se ha vuelto polémica, lo que dio lugar a que muchos profesionales de la salud dudaran en permitir la ingesta de alimentos y bebidas que contuvieran
ENC, incluso cuando los pacientes enfrentaban patologías como la obesidad y la diabetes.

A la luz de los datos toxicocinéticos de cada ENC, del margen de seguridad que brinda la IDA (con un factor de seguridad que es 100 veces menor que la máxima dosis diaria en la que no se aprecian efectos adversos) y considerando el bajo nivel de exposición a los ENC, esta preocupación sobre la seguridad de los ENC parecería ser desproporcionada.

\section{Conflicto de intereses:}

El autor ha recibido honorarios y pagos por consultoría de varias compañías biotecnológicas, farmacéuticas y de alimentos y bebidas. También ha recibido honorarios, pagos por consultoría y financiamiento de estudios de investigación sin restricción alguna, de fuentes gubernamentales y entidades no lucrativas. Ninguna de las entidades mencionadas tuvo ni tiene rol alguno en el diseño ni en la preparación del presente manuscrito.

\section{REFERENCIAS}

1. Organización Mundial de la Salud. Directriz: ingesta de azúcares para adultos y niños. Ginebra, Suiza: OMS; 2015. [Consulta: 8 de noviembre de 2017]. Disponible en: http: / / apps.who.int / iris / bitstream / 10665/154587 / 2 WHO_ NMH_NHD_15.2_spa.pdf.

2. Sugar projections: consumption per capita. OECD-FAO 2015:32. [Consulta: 8 de noviembre de 2017]. Disponible en: http:/ / www.oecd-ilibrary.org/agriculture-and-food/ oecd-fao-agricultural-outlook-2015/ sugar-projectionsconsumption-per-capita_agr_outlook-2015-table135-en.

3. Drewnowski A. Intense sweeteners and the control of appetite. Nutr Rev. 1995; 53(1):1-7.

4. Schmidt-Hebbel H. Avances en aditivos alimentarios y la reglamentación de los alimentos. Aplicaciones y comentarios de orden químico y tecnológico. Santiago de Chile: Universitaria; 1990.Págs.64-8.

5. Arnold DL, Krewski D, Munro IC. Saccharin: a toxicological and historical perspective. Toxicology. 1983; 27(3-4):179-256.

6. Klug C, von Rymon LipinskiGW. Acesulfame potassium. In O'Brien Nabors L (Ed.). Alternative Sweeteners. 4th ed. Boca Raton, FL: CRC Press; 2012.Págs.13-30.

7. Magnuson BA, Carakostas MC, Moore NH, et al. Biological fate of low-calorie sweeteners. Nutr Rev. 2016;74(11):670-89.

8. Prakash I, Chaturvedula VS. Structures of some novel a-glucosyl diterpene glycosides from the glycosylation of steviol glycosides. Molecules. 2014; 19(12):20280-94.

9. ButchkoHH,StargelWW, ComerCP, etal. Aspartame: review of safety. Regul Toxicol Pharmacol. 2002; 35(2 Pt 2):S1-93.

10. Sweatman TW, Renwick AG, Burgess CD. The pharmacokinetics of saccharin in man. Xenobiotica. 1981; 11(8):531-40.

11. Hutapea AM, Toskulkao C, Buddhasukh D, et al. Digestion of stevioside, a natural sweetener, by various digestive enzymes. J Clin Biochem Nutr. 1997; 23(3):177-86.

12. Gardana C, Simonetti P, Canzi E, et al. Metabolism of stevioside and rebaudioside A from Stevia rebaudiana extracts by human microflora. J Agric Food Chem. 2003; 51(22):6618-22. 
13. Koyama E, Kitazawa K, Ohori Y, et al. In vitro metabolism of the glycosidic sweeteners, stevia mixture and enzymatically modified stevia in human intestinal microflora. Food Chem Toxicol. 2003; 41(3):359-74.

14.Hooper NM, Hesp RJ, Tieku S. Metabolism of aspartame by human and pig intestinal microvillar peptidases. Biochem J. 1994; 298 Pt 3:635-9.

15. Stegink LD. Aspartame metabolism in humans: Acute dosing studies. In Stegink LD, Filer LJ Jr (Ed.). Aspartame: Physiology and Biochemistry. New York, NY: Marcel Dekker; 1984.Págs.509-53.

16. Sweatman TW, Renwick AG. The tissue distribution and pharmacokinetics of saccharin in the rat. Toxicol Appl Pharmacol. 1980; 55(1):18-31.

17. Renwick AG. The metabolism of intense sweeteners. Xenobiotica. 1986; 16(10-11):1057-71.

18. European Food Safety Authority. Scientific Opinion on the re-evaluation of aspartame (E 951) as a food additive. EFSA J. 2013; 11(12):3496.

19. Bender DA. The aromatic amino acids: phenylalanine, tyrosine and tryptophan. In: Amino Acid Metabolism. 3rd ed. Chichester, UK: Wiley-Blackwell; 2012.Págs.323-76.

20. Renwick AG. The intake of intense sweeteners - an update review. Food Addit Contam. 2006; 23(4):327-38.

21. Joint FAO/WHO Expert Committee on Food Additives. Steviol glycosides. In: Evaluation of Certain Food Additives. Geneva, Switzerland: World Health Organization; 2005. Págs.34-9.

22. Renwick AG. Intake of intense sweeteners. World Rev Nutr Diet. 1999; 85:178-200.

23. RenwickAG. Incidence and severity in relation to magnitude of intake above the ADI or TDI: use of critical effect data. Regul Toxicol Pharmacol. 1999; 30(2 Pt 2):S79-86.

24. RenwickAG. Toxicokineticsininfants and childrenin relation to the ADI and TDI. Food Addit Contam. 1998; 15(Suppl):17-35.

25. Renwick AG. Needs and methods for priority setting for estimating the intake of food additives. Food Addit Contam. 1996; 13(4):467-75.

26. ChattopadhyayS, RaychaudhuriU, Chakraborty R. Artificial sweeteners - a review. J Food Sci Technol. 2014; 51(4):611-21.

27. Joint FAO/WHO Expert Committee on Food Additives. 596: Acesulfame potassium. In Toxicological Evaluation of Certain Food Additives. Geneva, Switzerland: World Health Organization;1981.[Consulta:6 dejuniode2018]. Disponible en: http:/ / www.inchem.org/documents/jecfa/jecmono/ v16je02.htm.

28. US Food and Drug Administration. FDA statement on European aspartame study. Washington, DC., 2007. [Consulta: 8 de noviembre de 2017]. Disponible en: http:/ / www.fda.gov/Food/IngredientsPackagingLabeling/ FoodAdditivesIngredients/ucm208580.htm.

29. Prakash I, Chaturvedula VS, Markosyan A. Isolation, characterization and sensory evaluation of a hexa beta-Dglucopyranosyl diterpene from Stevia rebaudiana. Nat Prod Commun. 2013; 8(11):1523-6.

30. Joint FAO/WHO Expert Committee on Food Additives. Compendium of Food Additive Specifications. Rome, Italy: Food and Agriculture Organization of the United Nations;
2010. [Consulta: 6 de junio de 2018]. Disponible en: www. fao.org/docrep/013/i1782e/i1782e.pdf.

31. Carakostas MC, Curry LL, Boileau AC, BrusickDJ.Overview: the history, technical function and safety of rebaudioside A, a naturally occurring steviol glycoside, for use in food and beverages. Food Chem Toxicol. 2008; 46(Suppl 7):S1-10.

32. Wheeler A, Boileau AC, Winkler PC, et al. Pharmacokinetics of rebaudioside $\mathrm{A}$ and stevioside after single oral doses in healthy men. Food Chem Toxicol. 2008; 46(Suppl 7):S54-60.

33. Wingard RE Jr, Brown JP, Enderlin FE, et al. Intestinal degradation and absorption of the glycosidic sweeteners stevioside and rebaudioside A. Experientia. 1980; 36(5):51920.

34. Geuns JMC, Buyse J, Vankeirsbilck A, et al. Identification of steviol glucuronide in human urine. J Agric Food Chem. 2006; 54(7):2794-8.

35. Joint FAO/WHO Expert Committee on Food Additives. 720: Acesulfame potassium. In Toxicological Evaluation of Certain Food Additives. Geneva, Switzerland: World Health Organization; 1991. [Consulta:6dejuniode2018].Disponible en: http://www.inchem.org/documents/jecfa/jecmono/ v28je13.htm.

36. Sylvetsky AC, Gardner AL, Bauman V, et al. Nonnutritive sweeteners in breast milk. J Toxicol Environ Health A. 2015; 78(16):1029-32.

37. Gougeon R, Spidel M, Lee K, et al. Canadian Diabetes Association National Nutrition Committee Technical Review: Non-nutritive intense sweeteners in diabetes management. Can J Diab. 2004; 28(4):385-99.

38. Calzada-León R, Ruiz-Reyes M, Altamirano-BustamanteN, Padrón-Martínez MM. Características de losedulcorantes no calóricos y su uso en niños. Acta Pediatr Mex. 2013;34(3):14153.

39. Durán Agüero S, Salazar C, Espinoza J, Fuentealba F. ¿Se pueden recomendar en el embarazo los edulcorantes no nutritivos? Rev Chil Nutr. 2017; 44(1):103-10.

40. Stegink LD, Filer LJ, Baker GL, McDonnell JE. Effect of aspartame loading upon plasma and erythrocyte amino acid levels in phenylketonuric heterozygotes and normal adult subjects. J Nutr. 1979; 109(4):708-17.

41. Stegink LD, Filer LJ Jr, Baker GL, McDonnell JE. Effect of an abuse dose of aspartame upon plasma and erythrocyte levels of amino acids in phenylketonuric heterozygous and normal adults. J Nutr. 1980; 110(11):2216-24.

42. Caballero B, Mahon BE, Rohr FJ, et al. Plasma amino acid levels after single-dose aspartame consumption in phenylketonuria, mild hyperphenylalaninemia, and heterozygous state for phenylketonuria. J Pediatr. 1986; 109(4):668-71.

43. European Food Safety Authority. EFSA completes full risk assessment on aspartame and concludes it is safe at current levels of exposure. 2013. [Consulta: 16 de mayo de 2018]. Disponible en: https://www.efsa.europa.eu/en/press/ news/131210.

44. Administración Nacional de Medicamentos, Alimentos y Tecnología Médica. Acerca delosedulcorantes que contienen aspartamo. [Consulta: 16 de mayo de 2018]. Disponible en: http: / / www.anmat.gov.ar/Alimentos/Aspartamo.pdf. 